\title{
BMJ Open ARCADIA study protocol: a phase II, randomised, double-blind, placebo- controlled clinical trial to assess the safety and efficacy of AZD1656 in patients with diabetes hospitalised with suspected or confirmed COVID-19
}

Kieran McCafferty (D) , ${ }^{1}$ Zoe Hollowood, ${ }^{2}$ Michelle Allan, ${ }^{1}$ Donna Lockhart, ${ }^{2}$ Jamie Chorlton, ${ }^{2}$ John Martin ${ }^{2,3}$

To cite: McCafferty $\mathrm{K}$, Hollowood Z, Allan M, et al. ARCADIA study protocol: a phase II, randomised, doubleblind, placebo-controlled clinical trial to assess the safety and efficacy of AZD1656 in patients with diabetes hospitalised with suspected or confirmed COVID-19. BMJ Open 2021;11:e049650. doi:10.1136/ bmjopen-2021-049650

- Prepublication history and additional supplemental material for this paper are available online. To view these files, please visit the journal online (http://dx.doi.org/10.1136/ bmjopen-2021-049650).

Received 02 February 2021 Accepted 26 October 2021

Check for updates

(c) Author(s) (or their employer(s)) 2021. Re-use permitted under CC BY-NC. No commercial re-use. See rights and permissions. Published by BMJ.

${ }^{1}$ Department of Nephrology, Barts Health NHS Trust, London, UK

${ }^{2}$ St George Street Capital,

London, UK

${ }^{3}$ Division of Medicine, University College London, London, UK

Correspondence to

Dr Kieran McCafferty;

kieran.mccafferty4@nhs.net

\section{ABSTRACT}

Introduction COVID-19, caused by SARS-CoV-2, remains a global pandemic that has affected more than 100 million people worldwide with over 4.8 million deaths as of October 2021. Patients with diabetes have both an increased susceptibility to SARS-CoV-2 infection, enhanced disease severity and increased risk of mortality. The challenge presented in these patients is both to improve glycaemic control—which itself may confer a survival advantage - and to help maintain or restore immunological homeostasis. The specific glucokinase activator AZD1656 may address both of these challenges via its glucose-lowering effect and its immunological mechanism of action. The aim of the Alleviation of cardioRespiratory complications in patients with COVID-19 And DIAbetes (ARCADIA) trial is to investigate this hypothesis and determine whether AZD1656 can improve clinical outcomes for these patients.

Methods and analysis ARCADIA is a double-blind, placebo-controlled, interventional study of AZD1656 in 150 patients with either type 1 or type 2 diabetes who have been admitted to hospital with COVID-19. Eligible, consented patients will be randomised in a 1:1 manner to receive either active drug or matched placebo tablets while they are in hospital. All patients will receive the usual and current standard of care for patients with COVID-19 in that hospital. Clinical and laboratory data will be collected and assessed at baseline and throughout their participation in the study. Data will be captured in the case report form, which will be electronically archived at the end of the trial in the trial master file. The WHO 8-point Ordinal Scale for Clinical Improvement will be used to measure clinical outcome for the primary endpoint of the trial.

Ethics and dissemination Ethical approval has been obtained from the East Midlands-Leicester South Ethics Committee (REC 20/EM/0198) in the UK, from the National Bioethics Committee of Medicines and Medical Devices in Bucharest, Romania, and from the Ethics Committee IKEM a TN in Prague, Czech Republic. All study-related data will be used by the sponsor in accordance with local data protection law. In the UK, all patient identifiable data will
Strengths and limitations of this study

- The WHO 8-point Ordinal Scale for Clinical Improvement is a robust and clinically meaningful endpoint.

- The patient group represents a comorbid group with poorer outcomes compared with the general population necessitating additional therapeutic strategies.

- Additional therapies may be adopted as standard of care altering the event rate of the primary outcome, risking the study being underpowered.

- The short-term nature of the study limits any ability to examine the effects on 'Long COVID' syndrome.

- If the study is successful, the hypoglycaemic effect of this drug may limit its potential to be used in patients without diabetes.

be stored on a password-protected National Health Service N3 network with full audit trail. Anonymised data will be stored in an IS027001 certificated data warehouse. Trial registration number EudraCT 2020-002211-21, NCT04516759.

\section{INTRODUCTION}

Since its emergence almost 2years ago in December 2019, the novel coronavirus SARS-CoV-2 has caused almost 100 million cases of COVID-19 worldwide and has led to the death of 4.8 million people as of 12 October 2021. ${ }^{1}$ From the early stages of the pandemic, pre-existing diabetes has been identified as an independent risk factor for increased COVID-19-associated mortality. ${ }^{2}$ Subsequent work has confirmed that both type 1 (T1DM) and type 2 diabetes mellitus (T2DM) confer an increased risk of mortality, even with adjustment for age; moreover, poor glycaemic control (as defined by an elevated 
HbAlc level) was also identified as an independent predictor of increased mortality. ${ }^{3-5}$ A retrospective cohort study demonstrated that people with pre-existing diabetes required more intensive interventions including ventilatory support; it also observed that good glycaemic control as an inpatient was associated with a reduced need for medical intervention and improved mortality. ${ }^{6}$ COVID-19 may impact also on diabetic control leading to hyperglycaemia and diabetic ketoacidosis (DKA) which is a serious medical emergency associated with inpatient mortality. ${ }^{78}$

There are several potential explanations for the increased severity of COVID-19 seen in people with T1DM and T2DM with hyperglycaemia.

- Elevated glucose levels have been shown to increase SARS-CoV-2 viral load in human monocytes; once infected, glycolysis then sustains viral replication and the production of cytokines, driving T-cell dysfunction and epithelial cell death. ${ }^{9}$

- Infection with COVID-19 drives an inflammatory cytokine response, generation of reactive oxygen species and activation of the renin-angiotensin aldosterone system, thus COVID-19 infection exacerbates insulin resistance, hyperglycaemia and vascular endothelial damage. ${ }^{10}$

- In addition to the likely detrimental effects of T2DM and hyperglycaemia on COVID-19 infection outcomes, coronaviruses have also been shown to damage islet cells which can lead to acute hyperglycaemia via binding to ACE2 on pancreatic islet cells. Furthermore, interleukin 6 (IL-6) production as a key component of the cytokine storm associated with COVID-19 is a driver of ketogenesis and is elevated in DKA. $^{11}$

- T2DM represents a dysregulated immune state with a key pathological role for activated T lymphocytes. An imbalance between the proinflammatory Th1 and Th17 subtypes and the anti-inflammatory Th2 and Treg subtypes has been postulated to underlie both the pathology of the key features of T2DM itself and to be responsible for the development of diabetic complications such as nephropathy. ${ }^{12}$

The challenge presented by COVID-19 infection in people with pre-existing diabetes is therefore twofold: first, to improve glycaemic control, which in itself may confer a survival advantage, and second, to intervene in a dysfunctional immune system in order to improve outcomes. The current therapy of choice for glycaemic control of diabetic inpatients with COVID-19 is insulin. Concern has been raised whether alternative glucoselowering therapies might have unintended effects on the pathogenesis of COVID-19 due to an overlap in their function as antidiabetic medications with their role in the immune system: for example, dipeptidyl peptidase 4 (DPP-4) is known to have a role in inflammation and a cohort study examining correlation of disease outcome with pharmacotherapies suggested that patients on DPP-4 inhibitors were more likely to require intensive care unit admission. ${ }^{13}$ The use of Sodium-glucose co-transporter-2
(SGLT-2) inhibitors has been advised against due to the risk of precipitating DKA. ${ }^{14}$

We propose the use of a glucokinase activator (GKA) AZD1656. In keeping with other drugs of its class, AZD1656 activates glucokinase in pancreatic beta cells at the rate-limiting step in the glucose-stimulated secretion of insulin, and in hepatocytes to increase hepatic glucose uptake and glycogen synthesis leading to a reduction in blood glucose levels. Twenty-five clinical studies have been completed to evaluate the safety, tolerability, pharmacokinetics (PK), pharmacodynamics and drug interactions with AZD1656. Approximately 960 subjects have been exposed to AZD1656 in these clinical phase I and phase IIb studies, as part of a global development programme by AstraZeneca. AZD1656 has been shown to be a welltolerated medication in patients with T2DM and no safety concerns were raised in studies up to 6 months following the end of dosing. However, the antidiabetic effect of AZD1656 was found to cease after several months, thus limiting its long-term use for patients with diabetes. ${ }^{15} 16$

In addition to its glucose-lowering effect, AZD1656 may have additional benefits to patients with COVID-19 which involve its effects on immune function. ${ }^{17}$

In the immune response to infection, a delicate balance exists between T-cell activation to fight infection and excessive inflammation with cytokine release which is known to cause damage to infected host cells. Regulatory $\mathrm{T}$ cells (Tregs) are important in maintaining this balance.

Migration of activated Tregs to inflamed tissue is crucial for their immune-modulatory function and role in tissue repair. Treg migration is a process which is regulated by glucokinase-dependent glycolysis. ${ }^{17}$ A specific GKA, such as AZD1656, could enhance Treg migratory capacity and may prevent the development of cardiorespiratory complications observed in hospitalised patients with COVID-19, leading to lower requirements for oxygen therapy and assisted ventilation, and reduced incidences of pneumonia and acute respiratory distress syndrome (ARDS). Such an immunomodulatory effect might also reduce the likelihood of developing Long COVID, which is thought, at least in part, to be immune mediated. However, the current study design will be unable to capture these data.

In vivo studies using Tregs as an interventional treatment through adoptive transfer have shown benefit in an acute lung injury model through mediating the resolution of lung injury. ${ }^{18}$ This research shows that resolution in experimental lung injury is an active process in which Tregs play an essential role. The ability of Tregs to suppress the innate immune response is an important component of this. ${ }^{19}$

In an IL-6-rich inflammatory microenvironment, which is known to be a feature of COVID-19 infection, inflammatory Th17 cells are enhanced while Tregs are suppressed. ${ }^{20}$ A therapeutic agent which can shift the Th17/Treg balance towards Tregs might reduce the amplificatory inflammatory loop and therefore reduce the uncontrolled inflammation which occurs in ARDS. In an observational study, Th17/Treg ratio was positively 


\begin{tabular}{|c|c|c|}
\hline Patient State & Descriptor & Score \\
\hline Uninfected & $\begin{array}{l}\text { No clinical or virological } \\
\text { evidence of infection }\end{array}$ & 0 \\
\hline \multirow[t]{2}{*}{ Ambulatory } & No limitation of activities & 1 \\
\hline & Limitation of activities & 2 \\
\hline \multirow[t]{2}{*}{ Hospitalised (Mild disease) } & $\begin{array}{l}\text { Hospitalised, no oxygen } \\
\text { therapy required }\end{array}$ & 3 \\
\hline & $\begin{array}{l}\text { Hospitalised, oxygen therapy } \\
\text { by mask or nasal prongs }\end{array}$ & 4 \\
\hline \multirow[t]{3}{*}{$\begin{array}{l}\text { Hospitalised (Severe } \\
\text { disease) }\end{array}$} & $\begin{array}{l}\text { Non-invasive ventilation or } \\
\text { high flow oxygen }\end{array}$ & 5 \\
\hline & $\begin{array}{l}\text { Intubation and mechanical } \\
\text { ventilation }\end{array}$ & 6 \\
\hline & $\begin{array}{l}\text { Ventilation and additional } \\
\text { organ support - pressors, } \\
\text { RRT, ECMO }\end{array}$ & 7 \\
\hline Dead & Death & 8 \\
\hline
\end{tabular}

Figure 1 WHO Ordinal Scale for Clinical Improvement (COVID-19 trials, 18 February 2020). ECMO, Extracorporeal membrane oxygenation; RRT, Renal Replacement Therapy.

correlated with disease severity scores and 28-day mortality in a retrospective study of ARDS in $2015 .^{21}$ In addition, a recent study reported that 90-day survival for patients with ARDS was $83 \%$ for patients with Tregs/CD4+ percentage equal to or greater than $10.5 \%$, but only $41 \%$ for patients with Tregs/CD4+ percentage less than $10.5 \%(\mathrm{p}=0.01) .{ }^{22}$

AZD1656 is therefore a strong therapeutic candidate for use in patients with diabetes with COVID-19 who are admitted to hospital to reduce disease severity by reducing hyperglycaemia and also by potentially ameliorating the immune dysregulation by activating Tregs.

\section{METHODS AND ANALYSIS}

\section{Study objectives}

The primary objective of the study will be to determine the effect of AZD1656 on cardiorespiratory complications in hospitalised patients with diabetes with COVID-19, as measured using the WHO 8-point Ordinal Scale for Clinical Improvement (see figure 1). The secondary objectives will focus on glycaemic control, duration of hospitalisation and safety and tolerability. The exploratory objectives will include an assessment of the immunological effects of the drug and an assessment of whether AZD1656 affects the extent of any cardiac injury related to COVID-19 in hospitalised patients with diabetes. A complete list of the trial objectives is provided in table 1 .

\section{Study population and inclusion criteria}

The ARCADIA study is an interventional phase II, randomised, double-blind, placebo-controlled clinical trial to assess the safety and efficacy of AZD1656 in people with T1DM or T2DM hospitalised with suspected or confirmed COVID-19. Eligible patients will be randomised to receive either active drug or matched placebo on a 1:1
Table 1 Primary, secondary and exploratory objectives of ARCADIA (including measurement variables)

\begin{tabular}{|c|c|}
\hline Objectives & \\
\hline $\begin{array}{l}\text { Primary } \\
\text { objective }\end{array}$ & $\begin{array}{l}\text { To determine the effect of AZD1656 on the } \\
\text { cardiorespiratory complications of COVID-19 in } \\
\text { hospitalised patients with diabetes with known } \\
\text { or suspected COVID-19, as measured using } \\
\text { the WHO 8-point Ordinal Scale for Clinical } \\
\text { Improvement (see figure 1) compared with } \\
\text { placebo. }\end{array}$ \\
\hline $\begin{array}{l}\text { Secondary } \\
\text { objectives }\end{array}$ & $\begin{array}{l}\text { To assess the extent to which AZD1656 supports } \\
\text { maintenance of adequate glycaemic control in } \\
\text { hospitalised patients with diabetes with known or } \\
\text { suspected COVID-19. }\end{array}$ \\
\hline & $\begin{array}{l}\text { To assess the safety and tolerability of AZD1656 } \\
\text { in the management of diabetes in hospitalised } \\
\text { patients with diabetes with known or suspected } \\
\text { COVID-19. }\end{array}$ \\
\hline & $\begin{array}{l}\text { To determine whether AZD1656 affects duration } \\
\text { of hospital stay, requirement for mechanical } \\
\text { ventilation or mortality in patients with diabetes } \\
\text { with known or suspected COVID-19. }\end{array}$ \\
\hline $\begin{array}{l}\text { Exploratory } \\
\text { objectives }\end{array}$ & $\begin{array}{l}\text { To determine the pharmacokinetics (PK) of } \\
\text { AZD1656 in patients with diabetes with known or } \\
\text { suspected COVID-19. }\end{array}$ \\
\hline & $\begin{array}{l}\text { To explore the effects of AZD1656 on } \\
\text { immunophenotyping characteristics during } \\
\text { COVID-19 infection in hospitalised patients with } \\
\text { diabetes. }\end{array}$ \\
\hline & $\begin{array}{l}\text { To explore the effects of AZD1656 on } \\
\text { immunochemistry characteristics during COVID-19 } \\
\text { infection in hospitalised patients with diabetes. }\end{array}$ \\
\hline & $\begin{array}{l}\text { To explore whether AZD1656 affects the extent } \\
\text { of any cardiac injury related to COVID-19 in } \\
\text { hospitalised patients with diabetes. }\end{array}$ \\
\hline & $\begin{array}{l}\text { To explore if ethnicity affects the clinical outcome } \\
\text { of hospitalised patients with diabetes with known } \\
\text { or suspected COVID-19 treated with AZD1656 } \\
\text { versus placebo. }\end{array}$ \\
\hline & $\begin{array}{l}\text { To explore if 25-hydroxyvitamin D levels at } \\
\text { baseline affect the clinical outcome of hospitalised } \\
\text { patients with diabetes with known or suspected } \\
\text { COVID-19 treated with AZD1656 versus placebo. }\end{array}$ \\
\hline
\end{tabular}

${ }^{*}$ As measured by the need to increase baseline medication requirements or the need to add additional diabetic medications to maintain appropriate blood glucose levels.

basis. The study will be conducted in the UK, Romania and Czech Republic through the second half of 2020 and the first half of 2021. New sites were added during the trial as a strategy for ensuring enrolment completion. All inclusion and exclusion criteria are listed in box 1 .

\section{Informed consent and study governance}

For each study patient, written informed consent will be obtained prior to any protocol-related activities. Patients will give informed consent to the investigations set out in the schedule of activities, which are described in the patient information leaflet (see online supplemental files 1 and 2). This will include the opportunity to 'opt-in' to 


\section{Box 1 Inclusion and exclusion criteria}

Inclusion criteria

- Male or female.

- Aged 18 and older.

- Have either type 1 (T1DM) or type 2 diabetes mellitus (T2DM).

- Hospitalised with suspected or confirmed novel coronavirus (SARSCoV-2) infection, at the time of enrolment, categorised as stage 3, 4 or 5 on the WHO Ordinal Scale for Clinical Improvement.

- Blood glucose level at or above $4 \mathrm{mmol} / \mathrm{L}$.

- Able to take oral (tablet) formulation of medication.

- Patient is able to provide written informed consent prior to initiation of any study procedures.

Exclusion criteria

- In the opinion of the clinical team, progression to intubation or mechanical ventilation is imminent and inevitable, within the next 24 hours, irrespective of the provision of treatments.

- Patients admitted with primary suspected or proven Mycoplasma pneumoniae, Chlamydia pneumoniae and bacterial pneumonia, who acquired COVID-19 while hospitalised.

- Treatment with immunomodulators or antirejection drugs within the last 3 months.

- Pregnant or breast feeding.

- Men and women of childbearing potential, unwilling to use highly effective contraception during their participation in the trial and for 2 weeks after study completion.

- Anticipated transfer to another hospital which is not a study site within 72 hours.

- Known sensitivity to any of the study medication/placebo excipients.

- Prior dosing with AZD1656 on a previous clinical trial.

- Patients admitted as a result of and receiving immediate treatment for an acute asthmatic attack, acute myocardial infarction and acute cerebrovascular event.

- Any known non-COVID-19, non-diabetes-related, serious condition which, in the opinion of the clinical team, makes the patient unsuitable for the trial.

- Known history of drug or alcohol abuse within previous 12 months of screening.

- Known history of HIV, hepatitis C, unresolved hepatitis B or severe liver disease.

- Current or planned use of gemfibrozil or any other strong inhibitors of CYP2C8.

- Current or previous participation in another clinical trial where the patient has received a dose of an investigational medicinal product (IMP) containing small molecule treatment(s) within 30 days or five half lives (whichever is longer) prior to enrolment into this study or containing biological treatment(s) within 3 months prior to entry into this study.

an additional assessment of the impact of clotting factors on patient outcome.

The principal investigator (PI) retains overall responsibility for the informed consent of patients at their site and will ensure that any person delegated responsibility to participate in the informed consent process is duly authorised, trained and competent.

The ARCADIA trial will be overseen by the study management team established by the sponsor St George Street. A safety review committee (SRC) consisting of clinical and other experts will be established by the sponsor to review safety findings during the study and to help ensure patient safety. An SRC was selected over data monitoring committee on account of the study design, which includes a short enrolment period and short ( $\leq 21$ day) 'inpatient' dosing period. Day-to-day operations will be managed by a full-service clinical research organisation (CRO) (Clinipace). The study will be conducted in compliance with Good Clinical Practice, the principles of the Declaration of Helsinki and in accordance with local legal and regulatory requirements. Quality and regulatory compliance during the trial will be verified via implementation of the sponsor's study-specific audit plan. All audits will be executed by a company that is independent of the investigators and sponsor.

\section{Study procedures}

Patients who provide informed consent and who meet all eligibility criteria will be randomised by the investigator to receive either AZD1656 tablets (at a dose of $100 \mathrm{mg}$ two times per day) or matched placebo tablets. The treatment allocation sequence is computer generated; patients will be centrally assigned to randomised treatment using an interactive response technology (IRT) system (Medidata Rave Randomization and Trial Supply Management). This is a double-blind trial; neither the patient, investigator, pharmacist nor study team will know which treatment group the patient has been assigned to. In case of an emergency, the investigator has the sole responsibility for determining if unblinding of a patient's treatment assignment is warranted and for performing the unblinding of that patient via the IRT system.

The study design is illustrated in figure 2.

Study procedures will be conducted within the hospital where the patient is being treated for COVID-19, as set out in table 2. A clinical score will be measured every day for each patient using the WHO 8-point Ordinal Scale for Clinical Improvement. Patients will remain on study treatment while their score is 3,4 or 5 up to a maximum of 21 days. Once a patient is well enough to be discharged (WHO score 1 or 2) or has progressed to the point that they require mechanical ventilation (WHO score 6 or 7 ), study treatment will be discontinued.

During the trial, blood samples including cardiac biomarkers will be collected for both safety, efficacy and PK analysis. Immunophenotyping samples will be collected for analysis at Queen Mary University of London. Immunochemistry samples will be collected for analysis at York Bioanalytical Solutions. In order to investigate the impact of clotting factors on patient outcome,

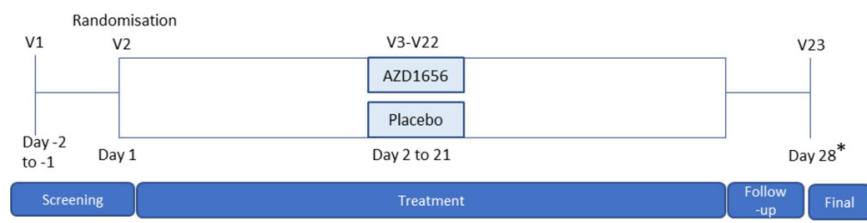

Figure 2 Study design. *Day 28 or day study end criteria are met. 


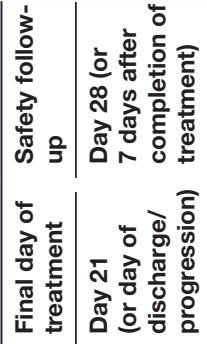

ลั่

좀으

ํํำ

ลิำ

ิํำ

สำ 오

बิ बิँ

वิ

ปิ

금

조을

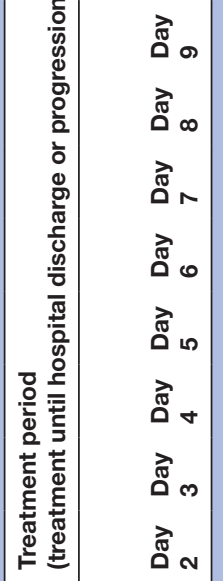
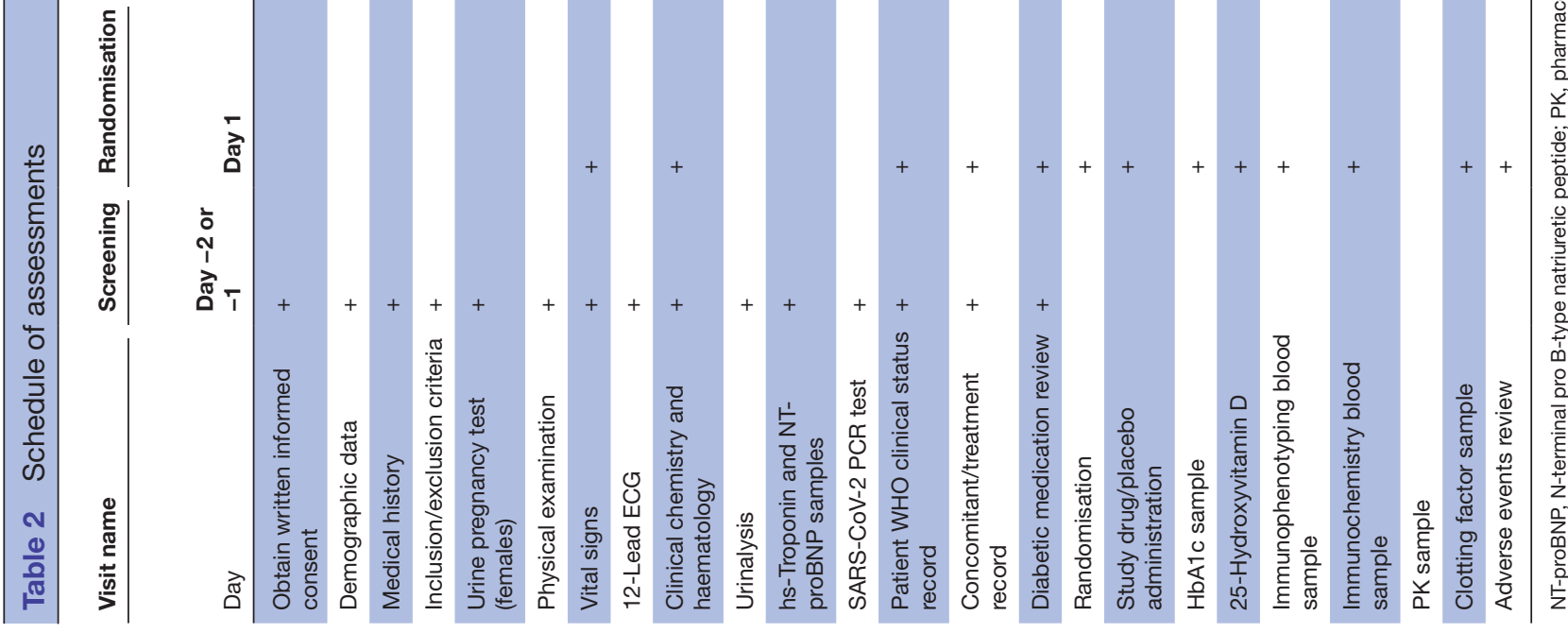
a small blood sample will be taken at baseline and on day 8 , and frozen on-site for future analysis.

Concomitant medications will be recorded, including any antidiabetic medications which may be adjusted at the discretion of the treating physician according to local practice. All adverse events, which the PI considers as not directly attributable to COVID-19 complications, will be reported for safety analysis.

When the study end criteria are met for a patient, assessments will be performed to collect all relevant outcome data. Patients will then have a final study visit 7 days after completion of study treatment to capture any new safety information or changes to concomitant medication, at which point they will be discharged from the study. This visit will be conducted in hospital if the patient has not been discharged, or via telephone call if the patient has been discharged. Patients who withdraw from the study after receiving treatment will not be replaced. The primary reason for a patient discontinuing treatment prematurely will be selected from the following standard categories: adverse event, intubation/mechanical ventilation, protocol violation, pregnancy, repeated events of minor hypoglycaemia or one event of major hypoglycaemia, introduction of strong inhibitors of CYP2C8 (prohibited medication) or other if the patient was terminated for a reason other than those listed above.

All data collected during the trial for each patient will be recorded in the patient's case report form which will be electronically archived at the end of the trial in the trial master file (TMF).

\section{Sample size}

Approximately 165 patients will be screened to achieve 150 randomly assigned patients to AZD1656 or placebo for an estimated total of 75 evaluable patients per group. Approximately 30 trial sites in the UK, Romania and Czech Republic will recruit the 150 randomised patients. (For a complete list of participating sites please visit www. clinicaltrials.gov.) A two-group $\chi^{2}$ test with a 5\% twosided significance level will have $76.74 \%$ power to detect the difference between a placebo proportion of 0.6 and an AZD1656 proportion of 0.8 when the sample size is 150 ( 75 /group). In the case that the portion of placebo patients meeting WHO categories 1-3 or live discharge at day 14 is higher (0.65), then the power to find a similar 20 percentage point increase would be $81.4 \%$.

\section{Study drug adherence and accountability}

The amount of study drug dispensed, administered to study patients, unused by study patients and the amount received from and returned to the sponsor will be documented at each investigational site throughout the course of the study to enable dosing adherence to be tracked and verified. Whole blood samples will also be collected for measurement of plasma concentrations of AZD1656 from each patient at three separate timepoints during the trial.

\section{Analysis}

Data generated during the trial will be managed according to the data management plan and analysed according to the statistical analysis plan (SAP). The full-analysis set (FAS) or intention-to-treat population includes all randomised patients who receive at least one dose of assigned treatment. The per-protocol set includes all patients of the FAS without major protocol deviations (and other criteria to be defined at a blinded data review meeting). Following the intent-to-treat principle, patients will be analysed as randomised. This will be the primary analysis set for primary and key secondary endpoints. The primary endpoint is the clinical improvement measured as the percentage of patients at day 14 who are in categories 1-3 according to the WHO 8-point Ordinal Scale for Clinical Improvement, comparing AZD1656 treatment to placebo. The PK analysis set includes all patients who received a single dose of AZD1656 and have at least one postdose PK measurement. Demographics and baseline characteristics will be analysed descriptively. Primary, key secondary and key exploratory efficacy parameters will be displayed descriptively, and appropriate tests are conducted on the treatment impact. To explore if subgroups affect the clinical outcome of hospitalised patients with diabetes treated with AZD1656 versus placebo, the primary efficacy analysis on clinical improvement will be repeated for subgroups of these variables: vitamin $\mathrm{D}$ group, race, sex, age group and diabetes type.

Missing data are assumed to be missing completely at random. Thus, they will not be imputed, and the data will be analysed as they are recorded. If a relevant proportion of missing data or data recorded after events interfering with the efficacy assessment are observed, additional sensitivity analyses will be performed. No interim analysis is planned. Full details of the planned statistical analyses will be described in the trial SAP.

\section{Collating and reporting the results}

This study will be registered in the EudraCT database and ClincalTrials.gov (first posted 18 August 2020) and study results will be posted in accordance with the applicable laws and regulations. A clinical study report will be developed at completion of data analysis according to International Conference on Harmonization E3 ('ICH E3) guidelines submitted to EudraCT in accordance with the required timelines according to the European Clinical Trials Directive 2001/20/EC. Results will also be submitted for publication in a peer-reviewed journal within 12 months of the clinical study report being finalised.

\section{Patient and public involvement}

Patients or the public were not directly involved in the conception, design and planning of this study.

\section{Ethics and dissemination}

Ethical approval has been obtained from the East Midlands-Leicester South Ethics Committee (REC 20/ $\mathrm{EM} / 0198$ ) in the UK, from the National Bioethics 
Committee of Medicines and Medical Devices in Bucharest, Romania, and from the Ethics Committee IKEM a TN in Prague, Czech Republic. Any amendments to the protocol will require independent ethics committee (IEC) and regulatory authority approval before implementation of changes made to the study design, except for changes necessary to eliminate an immediate hazard to study patients. All amendments will be communicated to the investigators and all other relevant parties via the project team following receipt of the necessary approvals.

On all study documentation, with the exception of the consent form and patient ID logs, patients will only be identified by their unique study identification code and will not be referred to by name, thus ensuring patient anonymity. All study-related data will be used by the sponsor in accordance with local data protection law. In the UK, all patient identifiable data will be stored on a password-protected National Health Service N3 network with full audit trail. Anonymised data will be stored in an ISO27001 certificated data warehouse.

The public liability insurance of the sponsor provides compensation for claims that arise in accordance with the regulatory requirements of the country involved, except for claims that arise from wilful misconduct or gross negligence. A copy of the insurance certificates will be held in the TMF and in the investigator site file.

On completion of the trial, all investigators will be provided with a copy of the final trial data set for the patients enrolled at their site. The clinical trial protocol, SAP and clinical study report synopsis will be disclosed once a manuscript relating to results is in a peer-reviewed medical journal. Deidentified individual participant data that underlie the published clinical trial results will also be made available to researchers who submit a methodologically sound research proposal to St George Street.

\section{DISCUSSION}

This multicentre, randomised, double-blind, placebocontrolled parallel group clinical trial examines the effect of AZD1656 versus placebo in people with diabetes who are admitted with proven or suspected COVID-19, in addition to the standard of care.

This cohort of patients with underlying diabetes was chosen because they represent a vulnerable group at higher risk of poor outcomes following admission with COVID-19. In addition, patients with diabetes frequently develop hyperglycaemia as a direct consequence of COVID-19 infection and also due to side effects of dexamethasone treatment. Therefore, this group of patients has specific unmet needs for therapies which help control hyperglycaemia while at the same time have an immunomodulatory effect on outcomes. AZD1656 was chosen because it has been shown in a number of studies to be safe and effective in the medium term for the control of hyperglycaemia as well as its immunomodulatory effect through its effects on enhancing Treg trafficking to sites of injury. Thus, this therapy offers the potential to prevent hyperglycaemia while also downregulating the inflammatory response from COVID-19.

A further strength of this trial is the choice of the primary endpoint. The WHO 8-point Ordinal Scale for Clinical Improvement was chosen as the primary endpoint as it is a robust reproducible clinically meaningful endpoint for patients. The scale ranging from discharge from hospital through to need for intubation and mechanical ventilation to death captures the broad spectrum of outcomes for patients admitted with COVID-19. We look forward to publishing the results of this study in a peer-reviewed, high-impact scientific journal.

\section{SUMMARY}

ARCADIA is the first trial to focus on treating both the glycaemic and immunological manifestations of COVID-19 in patients with T1DM and T2DM. This work will provide a valuable insight into the relationship between glucose control and COVID-19 clinical outcomes. It will also allow the potential immunomodulatory effects of this drug to be investigated. As an orally available tablet that is stable at room temperature, AZD1656 has the potential to become a global treatment option for patients with COVID-19.

\section{Limitations}

Despite this trial's many strengths, some limitations remain:

- As additional therapies are adopted as standard of care the event rate of the primary outcome may fall, risking the study being underpowered.

- While the proposed immunomodulatory effects of AZD1656 on Treg trafficking are independent of whether or not patients have diabetes, a cohort of patients with diabetes was chosen as this group could be more safely exposed to AZD1656 and was less likely to develop hypoglycaemia. However, this means that if this intervention is proven to be successful, we will be unable to extrapolate this to the non-diabetic population of patients with COVID-19 without subsequent studies.

- Given the treatment duration and short-term follow-up we will be unable to capture any long-term effects of AZD1656 on patient outcomes. Given the hypothesised effect of AZD1656 on inflammation resolution, it is possible that this AZD1656 treatment could lead to a reduction in the incidence or severity of the 'Long COVID' syndrome.

Correction notice This article has been corrected since it was first published. Author name has been corrected.

Acknowledgements We are grateful to Mr Martin Doerr of Clinipace who prepared the statistical plan and Professor Federica Marelli-Berg, BHF Professor of Cardiovascular Immunology and Centre Lead for Biochemical Pharmacology, Queen Mary University London, who designed the immunophenotyping panels for the study.

Contributors JC and JM conceived the study. JC, JM, KM and DL designed the study. Management of the trial and interpretation of data were overseen by JC. All authors (JC, KM, MA, JM, ZH, DL) provided advice on the final protocol. ZH and MA 
drafted the initial manuscript with input from JC, JM and KM. All authors approved the final manuscript.

Funding This non-commercial trial has been funded by St George Street Capital, the trial sponsor. Investment for the trial was sourced by Excalibur Medicines. Additional funding was provided by the UK government via the UK Research and Innovation (UKRI) 'Innovate UK' programme.

Competing interests None declared.

Patient consent for publication Obtained.

Provenance and peer review Not commissioned; externally peer reviewed.

Supplemental material This content has been supplied by the author(s). It has not been vetted by BMJ Publishing Group Limited (BMJ) and may not have been peer-reviewed. Any opinions or recommendations discussed are solely those of the author(s) and are not endorsed by BMJ. BMJ disclaims all liability and responsibility arising from any reliance placed on the content. Where the content includes any translated material, BMJ does not warrant the accuracy and reliability of the translations (including but not limited to local regulations, clinical guidelines, terminology, drug names and drug dosages), and is not responsible for any error and/or omissions arising from translation and adaptation or otherwise.

Open access This is an open access article distributed in accordance with the Creative Commons Attribution Non Commercial (CC BY-NC 4.0) license, which permits others to distribute, remix, adapt, build upon this work non-commercially, and license their derivative works on different terms, provided the original work is properly cited, appropriate credit is given, any changes made indicated, and the use is non-commercial. See: http://creativecommons.org/licenses/by-nc/4.0/.

ORCID iD

Kieran McCafferty http://orcid.org/0000-0002-0762-2270

\section{REFERENCES}

1 World Health Organisation. COVID-19 dashboard. Available: https:// cvid19.who.int; [Accessed 12 Oct 2021].

2 Zhou F, Yu T, Du R, et al. Clinical course and risk factors for mortality of adult inpatients with COVID-19 in Wuhan, China: a retrospective cohort study. Lancet 2020;395:1054-62.

3 Williamson EJ, Walker AJ, Bhaskaran K, et al. Factors associated with COVID-19-related death using openSAFELY. Nature 2020;584:430-6.

4 Barron E, Bakhai C, Kar P, et al. Associations of type 1 and type 2 diabetes with COVID-19-related mortality in England: a wholepopulation study. Lancet Diabetes Endocrinol 2020;8:813-22.

5 Holman N, Knighton P, Kar P, et al. Risk factors for COVID-19-related mortality in people with type 1 and type 2 diabetes in England: a population-based cohort study. Lancet Diabetes Endocrinol 2020;8:823-33.
6 Zhu L, She Z-G, Cheng X, et al. Association of blood glucose control and outcomes in patients with COVID-19 and pre-existing type 2 diabetes. Cell Metab 2020;31:1068-77.

7 Kulcsar KA, Coleman CM, Beck SE, et al. Comorbid diabetes results in immune dysregulation and enhanced disease severity following MERS-CoV infection. JCI Insight 2019;4:e131774.

8 Gibb FW, Teoh WL, Graham J, et al. Risk of death following admission to a UK hospital with diabetic ketoacidosis. Diabetologia 2016;59:2082-7.

9 Codo AC, Davanzo GG, Monteiro LdeB, et al. Elevated glucose levels favor SARS-CoV-2 infection and monocyte response through a HIF1 $\alpha /$ Glycolysis-Dependent axis. Cell Metab 2020;32:437-46.

10 Lim S, Bae JH, Kwon HS. COVID-19 and diabetes mellitus: from pathophysiology to clinical management. Nat Rev Endocrinol 2020:1-20.

11 Pal R, Banerjee M, Yadav U, et al. Clinical profile and outcomes in COVID-19 patients with diabetic ketoacidosis: a systematic review of literature. Diabetes Metab Syndr 2020;14:1563-9.

12 Xia C, Rao X, Zhong J. Role of T lymphocytes in type 2 diabetes and diabetes-associated inflammation. J Diabetes Res 2017;2017:1-6.

13 Dalan R, Ang LW, WYT T. The association of hypertension and diabetes pharmacotherapy with COVID-19 severity and immune signatures: an observational study. Eur Heart $J$ Cardiovasc Pharmacother 2020:pvaa098.

14 Musso G, Saba F, Cassader M, et al. Diabetic ketoacidosis with SGLT2 inhibitors. BMJ 2020;371:m4147.

15 Kiyosue A, Hayashi N, Komori H, et al. Dose-Ranging study with the glucokinase activator AZD1656 as monotherapy in Japanese patients with type 2 diabetes mellitus. Diabetes Obes Metab 2013;15:923-30.

16 Wilding JPH, Leonsson-Zachrisson M, Wessman C, et al. DoseRanging study with the glucokinase activator AZD1656 in patients with type 2 diabetes mellitus on metformin. Diabetes Obes Metab 2013;15:750-9.

17 Kishore M, Cheung KCP, Fu H. Regulatory T cell migration is dependent on glucokinase-mediated glycolysis. Immunity 2017;47:875-89.

18 D'Alessio FR, Tsushima K, Aggarwal NR, et al. Cd4+Cd25+Foxp3+ tregs resolve experimental lung injury in mice and are present in humans with acute lung injury. J Clin Invest 2009;119:2898-913.

19 Pietropaoli A, Georas SN. Resolving lung injury: a new role for Tregs in controlling the innate immune response. $J$ Clin Invest 2009;119:2891-4.

20 Chen N, Zhou M, Dong X, et al. Epidemiological and clinical characteristics of 99 cases of 2019 novel coronavirus pneumonia in Wuhan, China: a descriptive study. Lancet 2020;395:507-13.

21 Yu Z-xin, Ji M-sen, Yan J, et al. The ratio of Th17/Treg cells as a risk indicator in early acute respiratory distress syndrome. Crit Care 2015;19:82

22 Halter S, Aimade L, Barbié M, et al. T regulatory cells activation and distribution are modified in critically ill patients with acute respiratory distress syndrome: a prospective single-centre observational study. Anaesth Crit Care Pain Med 2020;39:35-44. 